\title{
Intraoperative and postoperative complications during microlaryngeal surgery
}

\author{
Marinov Ts. ${ }^{1}$, M. Belitova', T. Popov' ${ }^{2}$ N. Nizamova², J. Rangachev², D. Popova² \\ 'Department of Anesthesiology and Intensive Care; University Hospital "Queen Giovanna" - ISUL \\ Medical University - Sofia \\ ${ }^{2}$ Department of ENT Surgery; University Hospital "Queen Giovanna" - ISUL \\ Medical University - Sofia
}

\section{Abstract}

Theoretical basis: Patients presenting for microlaryngeal surgery may have a variety of comorbidities contributing to their voice symptoms and affecting anesthetic management.

Results: Many patients presenting for laryngeal surgery have a long history of heavy smoking and drinking. Many patients who present for laryngeal surgery are elderly and have cardiovascular disease. The rate of difficult endotracheal intubation may reach almost $16 \%$ among patients presenting for ear, nose, or throat cancer surgery, which is on average six times higher than among the general surgical patient population. Loss of the airway on induction, requiring emergent cricothyrotomy or tracheostomy, can be sudden, especially in patients with critical airway obstruction. Prospective trials identify the incidence of dental trauma after suspension laryngoscopy at $0 \%$ to $6.5 \%$, depending on the operator's experience, methodology of the study, dental injury criteria, preexisting dentition status of the patient, and suspension technique used. Minor surgical complications, such as sore throat, mucosal injury (e.g., cuts, edema, hematoma), and cranial nerve dysfunction (e.g., lingual, glossopharyngeal, hypoglossal), are most commonly observed. The risk of postoperative airway compromise is significantly greater among the patients who underwent diagnostic laryngoscopy than those in the general surgical population. Conclusion: Patients presenting for microlaryngeal surgery may have a variety of intraoperative and postoperative complications.

Key words: Microlaryngeal surgery, intraoperative complications, postoperative complications.
\end{abstract}

\section{Literature review}

Patients presenting for microlaryngeal surgery may have a variety of comorbidities contributing to their voice symptoms and affecting anesthetic management. Almost one half of the patients presenting with laryngeal and voice disorders have silent laryngopharyngeal reflux as the primary cause or as a significant etiologic factor. ${ }^{1}$ Coexistent significant glottic insufficiency (e.g., vocal cord paralysis) may place these patients at increased risk for aspiration of gastric contents. ${ }^{2}$

Many patients presenting for laryngeal surgery have a long history of heavy smoking and drinking, ${ }^{3}$ which are directly linked to the development of squamous cell carcinoma of the larynx, the second most common malignancy of the head and neck. It is not uncommon for these patients to present with anemia. ${ }^{4}$

Chronic cigarette smoking and alcohol use can cause induction of the cytochrome P450 multi-enzyme system, leading to increased perioperative requirements for opioids and neuromuscular blockers and generation of higher levels of potentially toxic metabolites of volatile halogenated anesthetic agents..$^{5-8}$ Patients with chronic alcohol consumption require preoperative evaluation of liver function and coagulation status. For those with advanced liver disease, controlled hypotensive techniques should be avoided, and intraoperative hypotension should be treated aggressively to prevent adverse outcomes associated with prolonged 
decrease in hepatic circulation and further deterioration of liver function. ${ }^{9}$

Many patients who present for laryngeal surgery are elderly and have cardiovascular disease.

The rate of difficult endotracheal intubation may reach almost $16 \%$ among patients presenting for ear, nose, or throat cancer surgery, which is on average six times higher than among the general surgical patient population. ${ }^{10-14}$ Comprehensive preoperative airway assessment is paramount; however, standard anesthesia airway assessment tests fail to account for aspiration risk, lower airway problems, and base of the tongue pathology (e.g., epiglottic cancer, epiglottic and vallecula cysts, lingual tonsillar hypertrophy). Pathology of the base of the tongue may be encountered with increased frequency in patients presenting for microlaryngeal surgery.

Postradiation changes in the neck and decreased mandibular protrusion are important factors predicting the risk of impossible mask ventilation, difficult mask ventilation, and difficult intubation in patients at risk for these conditions, ${ }^{15,16}$ and these risk factors may occur with increased frequency among patients presenting for microlaryngeal surgery. The pharyngeal space may also be reduced by limited submandibular compliance of the soft tissues (e.g., cancerous involvement, masses, inflammation, previous radiation therapy), which may result in difficult intubation or failed intubation due to the restriction of the space that accommodates the tongue during direct laryngoscopy. ${ }^{17}$

The incidence of complications during microlaryngeal surgery is small and is largely related to the experience of the anesthesiologist and the surgeon, as well as their cohesive team work, the characteristics of the patient population treated, and the status of the treating institution (e.g., academic, tertiary care, private practice). ${ }^{18-20}$

Microlaryngeal surgery remain very safe procedures. The mortality rate is exceedingly low $(0.02 \%$ to $0.6 \%){ }^{21}$ In a large, single-institution, retrospective review of 1093 endoscopic laryngeal surgery cases, Jaquet and colleagues ${ }^{22}$ reported no intraoperative deaths, an incidence of major complications of $0.37 \%$ (all related to barotrauma during subglottic JV), and no major complications for 281 pediatric patients between the ages of less than 1 year and 16 years.

Loss of the airway on induction, requiring emergent cricothyrotomy or tracheostomy, can be sud- den, especially in patients with critical airway obstruction. Proper preparation of the OR team is the key to promptly and efficiently dealing with intraoperative airway emergencies. An overall incidence of major barotrauma complications (e.g., cervicomediastinal emphysema, pneumothorax, tension pneumothorax $)$ is small $(0.2 \%$ to $0.5 \%)$ and these complications are most frequently observed during TTJV (1.1\%). ${ }^{22,23}$ Intraoperative bronchial or transbronchial biopsy represents an independent risk factor for intraoperative pneumothorax (about $10 \%$ ). These patients also carry a higher risk of developing pulmonary edema after transbronchial biopsy. ${ }^{19}$ Although the overall incidence of cardiovascular compromise may reach $20 \%$ to $50 \%,{ }^{24}$ major cardiac and cerebrovascular complications are rarely observed $(0 \%$ to $2.2 \%){ }^{19,22}$ Massive bleeding is rare and may be encountered in patients while coring out friable vascular tumors or during inadvertent perforation of the tracheal or bronchial wall with the laser or rigid bronchoscope. Ensuing respiratory failure with an inability to wean the patient from the ventilator has been described. The rigid bronchoscope should be used by experienced operators, because improper technique frequently results in dental or oropharyngeal trauma. Particular care must be exercised when introducing a rigid bronchoscope into the airway of patients considered at high risk for cervical spine (C-spine) dislocation during neck extension (e.g., elderly, patients with rheumatoid arthritis, those with congenital C-spine abnormalities). ${ }^{25}$ Prospective trials identify the incidence of dental trauma after suspension laryngoscopy at $0 \%$ to $6.5 \%$, depending on the operator's experience, methodology of the study, dental injury criteria, preexisting dentition status of the patient, and suspension technique used. ${ }^{26}$

Esophageal perforation is a rare event if flexible esophagoscopy is used. A significantly higher complication rate $(2.6 \%)$ was reported for rigid esophagoscopy, in which case patients with a history of head and neck cancer present a particular risk. ${ }^{18}$ Pulmonary aspiration remains a particular concern when the patient's airway is left unprotected (e.g., $\mathrm{JV}$ ), especially in patients at increased risk for aspiration of gastric contents. Intraoperative airway soiling may occur from aspiration of blood, secretions, surgical debris, or tumor cell contamination of the lower airway. ${ }^{27}$

Minor intraoperative anesthesia-related complications happen infrequently (2.6\%). The incidence of 
minor intraoperative laryngospasm during microlaryngeal surgery has been reported by Jaquet and colleagues as $3.1 \% .^{22}$ It was exclusively related to the use of AIV and was associated with a light plane of anesthesia. In contrast, prospectively recorded, surgery-related minor complications are common $(37.5 \%$ to $73 \%)$. Minor surgical complications, such as sore throat, mucosal injury (e.g., cuts, edema, hematoma), and cranial nerve dysfunction (e.g., lingual, glossopharyngeal, hypoglossal), are most commonly observed. The latter are likely caused by direct pressure or stretch injury associated with laryngoscopy or suspension of the laryngoscope, and they are related to the size of the operating laryngoscope used and the duration of suspension. Presenting symptoms in the recovery room may include dysesthesia and taste alteration, swallowing problems, and deviation of the tip of the tongue..$^{19,20,26}$

The risk of postoperative airway compromise is significantly greater among the patients who underwent diagnostic laryngoscopy than those in the general surgical population. ${ }^{28}$ The residual effects of anesthetics, analgesics, and inadequately reversed NMB may further contribute to the development of hypoventilation, atelectasis, and poor mobilization of secretions in the early postoperative period. Airway surgery invariably produces a certain degree of traumatic edema, which may precipitate acute airway obstruction postoperatively in an already compromised airway. Development of airway obstruction should be suspected if the patient has symptoms such as dyspnea, respiratory distress, and particularly inspiratory stridor. Aggressive early treatment with humidified oxygen and nebulized racemic epinephrine constitutes the first reasonable therapeutic intervention. Postoperative laryngospasm may precede or accompany airway obstruction and quickly lead to development of acute negative-pressure pulmonary edema. Although the incidence of these complications in the general surgical population is small $(0.3 \%$ and $0.09 \%$, respectively), negative-pressure pulmonary edema is preceded by laryngospasm in more than $50 \%$ of cases. Treatment is supportive and consists of reestablishment of airway patency, $\mathrm{O} 2$ supplementation, ventilatory support (i.e., CPAP or endotracheal intubation with positive-pressure ventilation and PEEP), management of fluid shifts, and maintenance of normal intravascular volume. ${ }^{29-31}$ Postoperative hemoptysis is usually associated with interventional bronchoscopy, for which the incidence may reach as high as $41 \%{ }^{19}$ It is common for the suspension laryngoscopy to aggravate preexisting temporomandibular joint disease, and these patients should be advised accordingly before surgery.

\section{References}

1. Falcone MT, Garrett CG, Slaughter JC, Vaezi M: Transnasal esophagoscopy findings: Interspecialty comparison. Otolaryngol Head Neck Surg 140:812-815, 2009.

2. Rubin AD, Sataloff RT: Vocal fold paresis and paralysis. Otolaryngol Clin North Am 40:1109-1131, 2007.

3. Rees L, Mason RA: Advanced upper airway obstruction in ENT surgery. Br J Anaesth CEPD Rev 2:134-138, 2002.

4. Xiao P, Zhang XS: Adult laryngotracheal surgery. Anesthesiol Clin 28:529-540, 2010.

5. Lieber CS: Ethanol metabolism, cirrhosis and alcoholism. Clin Chim Acta 257:59-84, 1997.

6. McKillop IH, Schrum LW: Alcohol and liver cancer. Alcohol 35:195-203, 2005.

7. Sweeney BP, Bromilow J: Liver enzyme induction and inhibition: Implications for anaesthesia. Anaesthesia 61:159-177, 2006.

8. Sweeney BP, Grayling M: Smoking and anaesthesia: The pharmacological implications. Anaesthesia 64:179-186, 2009.

9. Ziser A, Plevak DJ, Wiesner RH, et al: Morbidity and mortality in cirrhotic patients undergoing anesthesia and surgery. Anesthesiology 90:42-53, 1999 .

10. Arne J, Descoins P, Fusciardi J, et al: Preoperative assessment for difficult intubation in general and ENT surgery: Predictive value of a clinical multivariate risk index. Br J Anaesth 80:140-146, 1998.

11. Burkle CD, Walsh MT, Harrison BA, et al: Airway management after failure to intubate by direct laryngoscopy: Outcomes in a large teaching hospital. Can J Anaesth 52:634-640, 2005.

12. Rose DK, Cohen MM: The airway: Problems and predictions in 18,500 patients. Can J Anaesth 41:372-383, 1994.

13. Connelly NR, Ghandour K, Robbins L, et al: Management of unexpected difficult airway at a teaching institution over a 7-year period. J Clin Anesth 18:198-204, 2006.

14. Crosby ET, Cooper RM, Douglas MJ, et al: The unanticipated difficult airway with recommendations for management. Can J Anaesth 45:757-776, 1998 .

15. Kheterpal S, Han R, Tremper KK, et al: Incidence and predictors of difficult and impossible mask ventilation. Anesthesiology 105:885-891, 2006.

16. Kheterpal S, Martin L, Shanks AM, Tremper KK: Prediction and outcomes of impossible mask ventilation: A review of 50,000 anesthetics. Anesthesiology 110:891-897, 2009.

17. Bainton CR: Difficult intubation-what's the best test? Can J Anaesth 43:541-543, 1996.

18. Tsao GJ, Damrose EJ: Complications of esophagoscopy in an academic training program. Otolaryngol Head Neck Surg 142:500-504, 2010. 
19. Facciolongo N, Patelli M, Gasparini S, et al: Incidence of complica $\neg$ tions in bronchoscopy. Multicentre prospective study of 20,986 bronchoscopies. Monaldi Arch Chest Dis 71:8-14, 2009.

20. Corvo MA, Inacio A, Mello MB, et al: Extra-laryngeal complica $\neg$ tions of suspension laryngoscopy. Braz J Otorhinolaryngol 73:727-732, 2007.

21. Robinson PM: Complications of microlaryngeal surgery. Clin Oto $\neg$ laryngol Allied Sci 14:545-549, 1989.

22. Jaquet Y, Monnier P, Van Melle G, et al: Complications of different ventilation strategies in endoscopic laryngeal surgery: A 10-year review. Anesthesiology 104:52-59, 2006.

23. Shikowitz MJ, Abramson AL, Liberatore L: Endolaryngeal jet ven $\neg$ tilation: A 10-year review. Laryngoscope 101:455-461, 1991.

24. Yung KC, Courey MS: The effect of office-based flexible endo $\neg$ scopic surgery on hemodynamic stability. Laryngoscope 120:2231-2236, 2010.

25. Theodore PR: Emergent management of malignancy-related acute airway obstruction. Emerg Med Clin North Am 27:231-241, 2009.

26. Rosen CA, Andrade Filho PA, Scheffel L, Buckmire R: Oropharyngeal complications of suspension laryngoscopy: A prospective study. Laryngoscope 115:1681-1684, 2005.

27. Benjamin B, Lindholm CE: Systematic direct laryngoscopy: The Lindholm laryngoscopes. Ann Otol Rhinol Laryngol 112(Pt 1):787-797, 2003.

28. Hill RS, Koltai PJ, Parnes SM: Airway complications from laryn $\neg$ goscopy and panendoscopy. Ann Otol Rhinol Laryngol 96:691-694, 1987.

29. Westreich R, Sampson I, Shaari CM, et al: Negative-pressure pulmonary edema after routine septorhinoplasty: Discussion of pathophysiology, treatment, and prevention. Arch Facial Plast Surg 8:8-15, 2006.

30. Chuang YC, Wang CH, Lin YS: Negative pressure pulmonary edema: Report of three cases and review of the literature. Eur Arch Otorhinolaryngol 264:1113-1116, 2007.

31. Mamiya H, Ichinohe T, Kaneko Y: Negative pressure pulmonary edema after oral and maxillofacial surgery. Anesth Prog 56:49-52, 2009.

Reviewer: Prof. Dr. P. Benchev

\section{Correspondence to:}

Tsvetomir Marinov, MD, PhD

Department of Anesthesiology and Intensive Care

University Hospital "Queen Giovanna"- ISUL

Byalo More Str 8

Medical University - Sofia

E-mail: ts.marinov@abv.bg 